\title{
Correspondence
}

\section{Effusion after interpleural analgesia}

To the Editor:

Interpleural regional analgesia for postoperative pain relief is simple, safe and effective. ${ }^{-2}$ Pneumothorax is the most common complication. ${ }^{1-3}$

Following an elective cholecystectomy in a 29 -yr-old healthy human but before extubation of the trachea, the patient was turned to the left side, and an interpleural catheter was placed using the saline infusion technique. ${ }^{4}$ General anaesthesia was then discontinued, $20 \mathrm{ml}$ of $0.5 \%$ bupivicaine with epinephrine $\left(5 \mu \mathrm{g} \cdot \mathrm{ml}^{-1}\right)$ were injected and the patient had good pain relief. Nine hours later, when a top-up dose was needed, aspiration of the catheter yielded about $9 \mathrm{ml}$ of hazy, red-tinged fluid. This was sent for examination and the top-up dose was withheld. Overnight the patient received meperidine and promethazine for pain relief. Re-aspiration of the catheter the next day produced no aspirate and interpleural analgesia was continued for a further $48 \mathrm{hr}$ uneventfully using plain bupivicaine.

Examination of the aspirated fluid showed an RBC count of $60,660 \cdot \mu \mathrm{l}^{-1}$ and WBC count of $360 \cdot \mu \mathrm{l}^{-1}$ (differential leucocyte count from concentrated centrifuge was neutrophils $50 \%$, lymphocytes $13 \%$, monocytes $31 \%$, eosinophils $3 \%$ and bands $3 \%$ ).

The mechanism of this effusion is unclear. It could have been a reactionary pleural effusion to the catheter or to sodium metabisulphite and anhydrous citric acid which are added as antioxidants when epinephrine is added to bupivicaine. Subsequent use of plain bupivicaine produced no such effusion. Although no direct cause and effect relationship could be established from this case report, yet it highlights the need for vigilance.

\author{
Mohammed Maroof MD \\ Mahmood Siddique MD \\ Rashid M. Khan MD \\ Augustine Bonsu MD \\ Department of Anaesthesiology \\ King Fahad Hospital \\ P.O. Box 22490, Riyadh 11426 \\ Kingdom of Saudi Arabia
}

\section{REFERENCES}

1 Reiestad F, Stromskag KE. Interpleural catheter in the management of post-operative pain: a preliminary report. Reg Anesth 1986; 11: 89-91.

2 Reiestad F, Stromskag KE, Holmquist E. Interpleural administration of bupivicain in post-operative management of pain. Anesthesiology 1986; 65: A204.

3 Ananthanarayan $C$, Kashtan $H$. Pneumothorax after interpleural block in a spontaneously breathing patient. Anaesthesia 1990; 45: 342.

4 Scott PV. Interpleural regional analgesia: detection of the interpleural space by saline infusion. Br J Anaesth 1991; 66: 131-3.

\section{The laryngeal mask airway and fibreoptic laryngoscopy}

To the Editor:

Following a recent case of tracheal intubation using the Laryngeal Mask Airway ${ }^{\circledast 7}$ where other means had failed, ${ }^{1}$ we postulated that the Laryngeal Mask Airway might prove an effective means of holding the tongue forward and centrally positioning the fibreoptic bronchoscope during elective fibreoptic tracheal intubation.

The method currently used at our institution consists of fibreoptic bronchoscope insertion through a Williams Airway Intubator ${ }^{\text {wi }}$ with subsequent endotracheal tube insertion through this airway over the fibreoptic bronchoscope.

Patients who were scheduled to undergo elective surgery with general anaesthesia were selected and informed consent was obtained. The patients' airways were evaluated according to Mallampatti classification and general anaesthesia was induced. Either the Williams Airway Intubator ${ }^{\infty}$ or Laryngeal Mask Airway ${ }^{\infty}$ was inserted in random order, a fibreoptic bronchoscope was then directed to the laryngeal inlet, and the time to first view of the cords was recorded by an independent observer monitoring the procedure on a screen.

Our results suggest that the Laryngeal Mask Airway ${ }^{\text {(8) }}$ was as effective as the Williams Airway Intubator for quickly directing the fibreoptic bronchoscope to the vocal cords $(P-\mathrm{NS})$.

\begin{tabular}{lll}
\hline & $L M A$ & $T W A$ \\
\hline$n$ & 14 & 14 \\
Range (sec) & $3-45$ & $2-50$ \\
Mean (sec) & 8.9 & 12.9 \\
SD & 10.3 & 15.6 \\
\hline
\end{tabular}


We conclude that the Laryngeal Mask Airway ${ }^{\text {, with }}$ modification, would be as effective as the Williams Airway Intubator in fibreoptic laryngoscopy for the purpose of tracheal intubation.

The modifications which we would recommend are enlargement of the diameter of the Laryngeal Mask Airway ${ }^{2}$ so that it could accommodate a larger than $6 \mathrm{~mm}$ endotracheal tube and its shortening to facilitate removal of the Laryngeal Mask Airway over the endotracheal tube once the trachea has been intubated. ${ }^{2}$ We have used such a device with very good results and would encourage the manufacturer of the Laryngeal Mask Airway to look at modifying this very useful anaesthetic device ${ }^{3}$ for this purpose.

\section{Allison Crichlow MD \\ Rock Locken MD FRCPC \\ Joanne Todesco MD FRCPC \\ Department of Anaesthesia \\ Foothills Hospital \\ University of Calgary}

\section{REFERENCES}

1 Loken RG, Moir C. The Laryngeal Mask Airway as an aid to blind orotracheal intubation: use and removal (in press).

2 Brian AIF. Three cases of difficult intubation overcome by the Laryngeal Mask Airway. Anaesthesia 1985; 40: 353-5.

3 Maltby JR, Loken RG, Watson CN. Clinical appraisal of the Laryngeal Mask Airway. Can J Anaesth 1990; 37 : S108.

\section{Regurgitation and the laryngeal mask}

To the Editor:

Your editorial and the report by Nanji and Maltby ${ }^{1,2}$ on the laryngeal mask (LM) raise important isues.

The patient described was unsuitable for the LM. Gastric emptying is delayed by opiates and trauma, vomiting raises the suspicion of ileus and Alzheimer patients are unreliable historians.

The lack of published mortality with the LM after more than four years in the UK suggests it is safe to use in fasting patients and its role in the difficult airway is firmly established. ${ }^{3}$

However, we do need to reduce the chances of regurgitation, recognise its occurence and treat it appropriately. Instructions for reducing the risks in fasting patients are given in the Instruction Manual p 35, 39:4
$\mathrm{H} 2$ blockers and metoclopramide are wise in the obese. Gross obesity is normally a contra-indication to using the LM. Induction: Avoid etomidate and any agent known to be associated with vomiting. A small dose of droperidol is useful with short-acting opiates. Maintenance: Avoid inadequate anaesthesia. Recovery: Leaving the LM inflated and in place, maintain adequate anaesthesia until the patient is breathing spontaneously and positioned correctly for recovery.

\section{Recognising regurgitation}

Fluid may suddenly appear inside the LM tube. This should be watched for. The hypopharynx is filled by the inflated $\mathrm{LM}$, so that even $7-10 \mathrm{ml}$ is immediately apparent, giving early warning of regurgitation.

Straining during anaesthesia may produce high intraabdominal pressures, overcoming the limited resistance offered by the distal LM cuff which lies against the upper oesophageal sphincter. Gastric fluid may thus enter the mask interior where it can be aspirated during inspiration or blown up the LM tube during expiration. The glottis may close limiting the amount of aspiration but causing stridor.

\section{Treatment}

- Head down tilt

- Disconnect circuit to allow fluid to drain

- Reconnect circuit to give $100 \%$ oxygen

- Avoid forceful ventilation attempts

- Deliver small tidal volumes

- Deepen anaesthesia - iv propofol works well

- Perform suction through the LM (fibrescope is best), avoid high vacuum/prolonged suction.

Further treatment will depend on the extent of aspiration but chest $x$-ray is mandatory. Avoid removing or deflating the LM if possible - it is likely to be holding back more fluid. ${ }^{5}$ Laryngeal mask removal followed by paralysis and intubation create new hazards including loss of airway potency, obstruction by biting, further aspiration, loss of cough reflex and possible bronchospasm. Of four cases receiving the above treatment without intubation, none required admission to an intensive care unit. ${ }^{6}$

\section{A.I.J. Brain}

Northwick Park Hospital

London, England

\section{REFERENCES}

1 Fisher JA. Ananthanarayan $C$, Edelist $G$. Role of the laryngeal mask in airway management. Can $J$ Anaesth 1992; 39: 1-3.

2 Nanji GM, Maltby JR. Vomiting and aspiration 